PROCEEDINGS OF THE

AMERICAN MATHEMATICAL SOCIETY

Volume 132, Number 2, Pages 391-396

S 0002-9939(03)07017-5

Article electronically published on June 11, 2003

\title{
MARCINKIEWICZ'S THEOREM ON OPERATOR MULTIPLIERS OF FOURIER SERIES
}

\author{
MILUTIN R. DOSTANIĆ
}

(Communicated by Joseph A. Ball)

\begin{abstract}
We give some sufficient conditions on the operators $A_{m} \in \mathcal{B}\left(L^{p}(0,1)\right)$ which for each $\Phi_{m} \in L^{p}(0,1)$ imply the inequality$$
\int_{0}^{1} \int_{0}^{2 \pi}\left|\sum_{m} e^{i m x} \cdot A_{m} \Phi_{m}(y)\right|^{p} d x d y \leq c_{p}^{p} \int_{0}^{1} \int_{0}^{2 \pi}\left|\sum_{m} e^{i m x} \cdot \Phi_{m}(y)\right|^{p} d x d y,
$$$$
1<p<\infty \text {. }
$$

\section{INTRODUCTION}

Let $D$ denote the unit disc $\{z:|z|<1\}$ and let $d A(z)=d x d y(z=x+i y)$ be the Lebesgue measure on $D$. In investigations of boundedness of the integral operators on $L^{p}(w(|z|) d A(z))$ ( $w$ is weight function) it is often necessary to consider inequalities of the form

$$
\int_{0}^{1} \int_{0}^{2 \pi}\left|\sum_{m} e^{i m x} \cdot A_{m} \Phi_{m}(y)\right|^{p} d x d y \leq c_{p}^{p} \int_{0}^{1} \int_{0}^{2 \pi}\left|\sum_{m} e^{i m x} \cdot \Phi_{m}(y)\right|^{p} d x d y
$$

$\left(c_{p}\right.$ is a constant which depends only on $\left.p\right)$, where $A_{m} \in \mathcal{B}\left(L^{p}(0,1)\right)$ and $\Phi_{m} \in$ $L^{p}(0,1), m \in \mathbb{Z}$. Here $\mathcal{B}\left(L^{p}(0,1)\right)$ denotes the space of bounded operators on $L^{p}(0,1)$. For example, the problem of a precise two-sided estimate of the norm of the Cauchy operator on $L^{p}(D)$ reduces to an inequality of the form (1) with concrete operators $A_{m}$. Also, the problem of boundedness of the Bergman projection on $L^{p}(w(|z|) d A(z))$ (in terms of the weight $w$ ) reduces to an inequality of the form (1) and studying of the corresponding operators $A_{m}$. The results concerning the above-mentioned problems will appear in forthcoming papers.

For $A \in \mathcal{B}\left(L^{p}(0,1)\right),\|A\|_{\mathcal{B}\left(L^{p}(0,1)\right)}$ denotes its norm. On the other hand, when $A_{m}=\lambda_{m} I$ and $\Phi_{m} \equiv a_{m}$, inequality (1) becomes

$$
\int_{0}^{2 \pi}\left|\sum_{m} e^{i m x} \cdot \lambda_{m} a_{m}\right|^{p} d x \leq c_{p}^{p} \int_{0}^{2 \pi}\left|\sum_{m} e^{i m x} \cdot a_{m}\right|^{p} d x,
$$

which is precisely Marcinkiewicz's inequality for the multipliers of Fourier's series of functions from $L^{p}(0,2 \pi), 1<p<\infty$.

Received by the editors July 19, 2001 and, in revised form, September 20, 2002.

2000 Mathematics Subject Classification. Primary 42B15.

Key words and phrases. Marcinkiewicz's theorem, multipliers. 
Sufficient conditions for inequality (2) are given by Marcinkiewicz's theorem (see [4], pp. 346-348) on multipliers of Fourier's series:

(1) $\sup _{m \in \mathbb{Z}}\left|\lambda_{m}\right|<+\infty$

(2) $\sup _{m \geq 0} \sum_{|j|=2^{m}+1}^{2^{m+1}}\left|\lambda_{j}-\lambda_{j+1}\right|<+\infty$.

In this paper we will give some conditions for operators $A_{m} \in \mathcal{B}\left(L^{p}(0,1)\right)$, $m \in \mathbb{Z}$, which are sufficient for the inequality (1) to hold.

\section{Result}

Theorem 1. Let $\left(\lambda_{m}\right)_{m \in \mathbb{Z}}$ be a sequence of complex numbers such that

$$
\sup _{m \in \mathbb{Z}}\left|\lambda_{m}\right|<+\infty \quad \text { and } \quad \sup _{m \geq 0} \sum_{|j|=2^{m}+1}^{2^{m+1}}\left|\lambda_{j}-\lambda_{j+1}\right|<+\infty .
$$

If $\left(A_{m}\right)_{m \in \mathbb{Z}}$ is a sequence of operators in $\mathcal{B}\left(L^{p}(0,1)\right), 1<p<\infty$, such that

$$
\sum_{m \in \mathbb{Z}}\left\|\frac{A_{m}}{\lambda_{m}}-\frac{A_{m+1}}{\lambda_{m+1}}\right\|_{\mathcal{B}\left(L^{p}(0,1)\right)}<+\infty
$$

then there exists a constant $c_{p}$ (depending only on $p$ ) such that (1) holds for arbitrary functions $\Phi_{m} \in L^{p}(0,1)$. (All sums in (1) are finite, $x \in[0,2 \pi], y \in[0,1]$.)

Remark 1. If $A_{m}=\lambda_{m} I$ ( $I$ is a unit operator), then (4) is obviously satisfied and thus the previous theorem gives the inequality

$$
\int_{0}^{1} \int_{0}^{2 \pi}\left|\sum_{m} e^{i m x} \cdot \lambda_{m} \Phi_{m}(y)\right|^{p} d x d y \leq c_{p}^{p} \int_{0}^{1} \int_{0}^{2 \pi}\left|\sum_{m} e^{i m x} \cdot \Phi_{m}(y)\right|^{p} d x d y,
$$

which holds under assumption (3).

In particular, if $\Phi_{m}(y)=a_{m}$, we get Marcinkiewicz's theorem.

If $\lambda_{m}=0$ for $m \leq 0$ and $\lambda_{m}=1$ for $m \geq 1$, we get Riesz's Theorem on projection (see [1], pp. 113-117).

Before the proof the theorem we recall some facts about tensor products of normed spaces and tensor product of operators defined on these spaces (see e.g. [3] for more details).

Denote $E=L^{p}(0,2 \pi), F=L^{p}(0,1)(p \geq 1)$.

Let $E \otimes F$ denote the vector space generated by all functions of the form $u(x) v(y), u \in E, v \in F$ (tensor product). For

$$
z=\sum_{i=1}^{n} u_{i}(x) v_{i}(y) \in E \otimes F
$$

set

$$
\|z\|_{E \otimes F}=\inf \sum_{i}\left\|\alpha_{i}\right\|_{p}\left\|\beta_{i}\right\|_{p}
$$

where $\left\|\alpha_{i}\right\|_{p}=\left(\int_{0}^{2 \pi}\left|\alpha_{i}\right|^{p} d x\right)^{\frac{1}{p}}, \quad\left\|\beta_{i}\right\|_{p}=\left(\int_{0}^{1}\left|\beta_{i}\right|^{p} d y\right)^{\frac{1}{p}}$ and inf is taken over all possible (finite) representations of $z$ as $z=\sum_{i=1}^{n} \alpha_{i}(x) \beta_{i}(y), \alpha_{i} \in E, \beta_{i} \in F$. It 
is easy to check that $\|\cdot\|_{E \otimes F}$ is a norm on $E \otimes F$. Completing $E \otimes F$ with respect to this norm we get the Banach space $E \widehat{\otimes} F$. It is well known that $E \widehat{\otimes} F$ is isomorphic to $L^{p}((0,2 \pi) \times(0,1))$. This means that there exists a constant $c(p)$ such that

$$
(c(p))^{-1} \cdot\|z\|_{L^{p}((0,2 \pi) \times(0,1))} \leq\|z\|_{E \otimes F} \leq c(p) \cdot\|z\|_{L^{p}((0,2 \pi) \times(0,1))}
$$

for each $z \in E \otimes F$.

If $A \in \mathcal{B}\left(L^{p}(0,2 \pi)\right)$ and $B \in \mathcal{B}\left(L^{p}(0,1)\right)$ we define the operator $A \otimes B$ : $E \otimes F \longrightarrow E \otimes F$ as

$$
(A \otimes B)\left(\sum_{i=1}^{n} u_{i}(x) v_{i}(y)\right)=\sum_{i=1}^{n}\left(A u_{i}\right)(x) \cdot\left(B v_{i}\right)(y) .
$$

It is well known that

$$
\|A \otimes B\|_{\mathcal{B}(E \otimes F)}=\|A\|_{\mathcal{B}\left(L^{p}(0,2 \pi)\right)} \cdot\|B\|_{\mathcal{B}\left(L^{p}(0,1)\right)}
$$

and thus the operator $A \otimes B$ extends to the bounded operator on $E \widehat{\otimes} F$. We keep the same notation $A \otimes B$ for this extension.

Obviously

$$
\left\{\begin{array}{l}
A \otimes(B+C)=A \otimes B+A \otimes C, \\
(B+C) \otimes A=B \otimes A+C \otimes A .
\end{array}\right.
$$

Proof of Theorem 1. For $1<p<\infty$ let $P_{k}, k \in \mathbb{Z}$, be the linear operator on $L^{p}(0,2 \pi)$ defined by

$$
P_{k} f(x)=\frac{1}{2 \pi} \int_{0}^{2 \pi} f(y) e^{i k(x-y)} d y .
$$

Then the "scalar" Marcinkiewicz's theorem implies the existence of constant $c_{1}(p)$ such that

$$
\left\|\sum_{k=N_{1}}^{N_{2}} \lambda_{k} P_{k}\right\|_{\mathcal{B}\left(L^{p}(0,2 \pi)\right)} \leq c_{1}(p)
$$

$\left(N_{1}, N_{2}\right.$ are arbitrary integers, $c_{1}(p)$ a constant which depends only on $\left.p\right)$.

Consider the operator

$$
T=\sum_{m} P_{m} \otimes A_{m}
$$

We will show that $T$ is bounded on $E \widehat{\otimes} F$. To do this it is enough to show that it is bounded on $E \otimes F$.

Let $r \leq m \leq s, r, s \in \mathbb{Z}$. From the representation

$$
T=\sum_{m=r}^{s} \lambda_{m} P_{m} \otimes \frac{A_{m}}{\lambda_{m}},
$$

by putting

$$
S_{r, \nu}=\sum_{m=r}^{\nu} \lambda_{m} P_{m}, \quad \nu=r, r+1, \ldots, s,
$$

and using Abel's summation formula, we get

$$
T=S_{r, s} \otimes \frac{A_{s}}{\lambda_{s}}+\sum_{m=r}^{s-1} S_{r, m} \otimes\left(\frac{A_{m}}{\lambda_{m}}-\frac{A_{m+1}}{\lambda_{m+1}}\right) .
$$


From (17), (8), (44),(9) and the previous equality we obtain

$$
\|T\|_{\mathcal{B}(E \otimes F)} \leq c_{2}(p)
$$

where $c_{2}(p)$ is a constant depending on $p$ only. Note that if $\Phi_{m} \in L^{p}(0,1)$, then

$$
z=\sum_{m} e^{i m x} \Phi_{m}(y) \in E \otimes F
$$

and

$$
T z=\sum_{m} e^{i m x} A_{m} \Phi_{m}(y) \in E \otimes F .
$$

Hence, (10) gives

$$
\|T z\|_{E \otimes F} \leq c_{2}(p)\|z\|_{E \otimes F} .
$$

This inequality together with (6) yields

$$
\begin{aligned}
(c(p))^{-1}\|T z\|_{L^{p}((0,2 \pi) \times(0,1))} & \leq\|T z\|_{E \otimes F} \\
& \leq c_{2}(p)\|z\|_{E \otimes F} \leq c(p) c_{2}(p)\|z\|_{L^{p}((0,2 \pi) \times(0,1))},
\end{aligned}
$$

i.e.

$$
\|T z\|_{L^{p}((0,2 \pi) \times(0,1))} \leq c_{p}^{p}\|z\|_{L^{p}((0,2 \pi) \times(0,1))}
$$

where $c_{p}=\left((c(p))^{2} c_{2}(p)\right)^{\frac{1}{p}}$, which completes the proof.

Remark 2. Putting

$$
\Phi_{m}(y)=\left\{\begin{array}{cl}
r_{m}(t) f_{m}(y) ; & m \geq 1, f_{m} \in L^{p}(0,1), \\
0 ; & m \leq 0,
\end{array}\right.
$$

in (11) ( $r_{m}$ are Rademacher's functions) we get

$$
\int_{0}^{1} \int_{0}^{2 \pi}\left|\sum_{m \geq 1} e^{i m x} r_{m}(t) A_{m} f_{m}(y)\right|^{p} d x d y \leq c_{p}^{p} \int_{0}^{1} \int_{0}^{2 \pi}\left|\sum_{m \geq 1} e^{i m x} r_{m}(t) f_{m}(y)\right|^{p} d x d y,
$$

whence by integration on $t \in[0,1]$, and using Khinchin's inequality, we get

$$
\int_{0}^{1}\left(\sum_{m \geq 1}\left|A_{m} f_{m}(y)\right|^{2}\right)^{\frac{p}{2}} d y \leq d_{p}^{p} \int_{0}^{1}\left(\sum_{m \geq 1}\left|f_{m}(y)\right|^{2}\right)^{\frac{p}{2}} d y
$$

( $d_{p}$ a constant depending only on $p$ ). Therefore, if there holds (44), then we have (11). Inequality (11) was proved in [4, p. 336, in the case where all the operators $A_{m}$ are mutually equal. From Theorem 1 it follows that (11) holds in the case where $A_{m}$ need not be equal but are "mutually near" in the sense that there holds in (4). Now suppose that (1) holds when we replace (4) by the conditions analogous to Marcinkiewicz's conditions:

$$
\left\{\begin{array}{l}
\sup _{m \in \mathbb{Z}}\left\|A_{m}\right\|_{\mathcal{B}\left(L^{p}(0,1)\right)}<+\infty \\
\sup _{m \geq 0} \sum_{|j|=2^{m}+1}^{2^{m+1}}\left\|A_{j}-A_{j+1}\right\|_{\mathcal{B}\left(L^{p}(0,1)\right)}<+\infty
\end{array}\right.
$$

Then (11) holds as well. 
Let $B_{m} \in \mathcal{B}\left(L^{p}(0,1)\right)$ be a sequence of operators such that $\sup _{m \geq 1}\left\|B_{m}\right\|_{\mathcal{B}\left(L^{p}(0,1)\right)}<$ $+\infty$ and let

$$
A_{m}=\left\{\begin{array}{ccc}
0 & ; \quad m \neq 2^{k} \\
B_{k} \quad ; & m=2^{k} .
\end{array}\right.
$$

Clearly $\left(A_{m}\right)_{m=1}^{\infty}$ satisfies (12), so (11) holds for an arbitrary (finite) choice of functions $f_{m} \in L^{p}(0,1)$. Putting

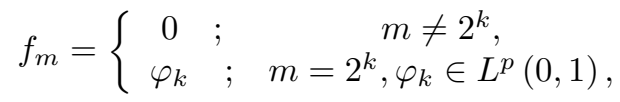

we get

$$
\int_{0}^{1}\left(\sum_{k \geq 1}\left|B_{k} \varphi_{k}(y)\right|^{2}\right)^{\frac{p}{2}} d y \leq d_{p}^{p} \int_{0}^{1}\left(\sum_{k \geq 1}\left|\varphi_{k}(y)\right|^{2}\right)^{\frac{p}{2}} d y,
$$

i.e. an inequality of the form (11) for which it is not clear whether it holds only under the assumption $\sup _{k \geq 1}\left\|B_{k}\right\|_{\mathcal{B}\left(L^{p}(0,1)\right)}<+\infty$.

If $A_{m}$ are integral operators on $L^{p}(0,1), 1<p<\infty$, then (44) can sometimes be replaced by the condition imposed on the kernel of $A_{m}$.

Namely we have the following

Corollary 1. Let $K_{m}(x, y), m \in \mathbb{Z}$, be homogeneous functions of order -1 and let $A_{m}^{\prime}$ be the bounded operators on $L^{p}(0, \infty)$ defined by

$$
A_{m}^{\prime} f(x)=\int_{0}^{\infty} K_{m}(x, y) f(y) d y
$$

such that

$$
\sum_{m} \int_{0}^{\infty} y^{-\frac{1}{p}}\left|\frac{K_{m}(1, y)}{\lambda_{m}}-\frac{K_{m+1}(1, y)}{\lambda_{m+1}}\right| d y<+\infty .
$$

If $A_{m}: L^{p}(0,1) \longrightarrow L^{p}(0,1)$ are the operators defined by

$$
A_{m} f(x)=\int_{0}^{1} K_{m}(x, y) f(y) d y
$$

then (1) holds.

Proof. Let $P: L^{p}(0, \infty) \longrightarrow L^{p}(0, \infty)$ be the linear operator defined by

$$
\operatorname{Pf}(x)=\mathcal{X}_{[0,1]}(x) f(x),
$$

where

$$
\mathcal{X}_{[0,1]}(x)= \begin{cases}1, & x \in[0,1] \\ 0, & x \notin[0,1]\end{cases}
$$

Applying the Hardy-Littlewood inequality (see [2]) we get

$$
\begin{aligned}
\left\|\frac{A_{m}}{\lambda_{m}}-\frac{A_{m+1}}{\lambda_{m+1}}\right\|_{\mathcal{B}\left(L^{p}(0,1)\right)} & =\left\|P\left(\frac{A_{m}^{\prime}}{\lambda_{m}}-\frac{A_{m+1}^{\prime}}{\lambda_{m+1}}\right) P\right\|_{\mathcal{B}\left(L^{p}(0, \infty)\right)} \\
& \leq\left\|\frac{A_{m}^{\prime}}{\lambda_{m}}-\frac{A_{m+1}^{\prime}}{\lambda_{m+1}}\right\|_{\mathcal{B}\left(L^{p}(0, \infty)\right)}
\end{aligned}
$$


and

$$
\left\|\frac{A_{m}^{\prime}}{\lambda_{m}}-\frac{A_{m+1}^{\prime}}{\lambda_{m+1}}\right\| \|_{\mathcal{B}\left(L^{p}(0, \infty)\right)} \leq \int_{0}^{\infty} y^{-\frac{1}{p}}\left|\frac{K_{m}(1, y)}{\lambda_{m}}-\frac{K_{m+1}(1, y)}{\lambda_{m+1}}\right| d y,
$$

which implies the corollary.

\section{ACKNOWLEDGMENT}

The author is grateful to the referee for the suggestion which helped to improve this article.

\section{REFERENCES}

[1] J. B. Garnett, "Bounded analytic functions", Pure and Applied Mathematics, vol. 96, Academic Press, 1981. MR 83g:30037

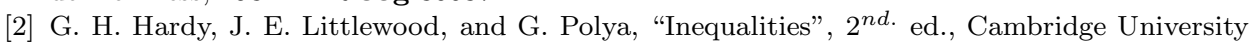
Press, Cambridge, UK, 1952. MR 13:727e

[3] R. Schatten, "A Theory of cross-spaces", Ann. Math. Studies, $\mathcal{N}^{\circ} 26,1950$. MR 12:186e

[4] A. Zygmund, "Trigonometric Series", Izdatelstvo "Mir", Moscow, 1965. MR 31:2554

Matematicki Fakultet, University of Belgrade, Studentski Trg 16, 11000 Belgrade, SERBIA

E-mail address: domi@matf.bg.ac.yu 